\title{
Towards understanding and acting on risk factors for developmental psychopathology
}

\author{
Carmen Moreno ${ }^{1}$
}

Published online: 30 January 2018

c) Springer-Verlag GmbH Germany, part of Springer Nature 2018

Most common mental disorders appear before adulthood [1]. Therefore, the ability to identify at-risk children is essential for the design and delivery of prevention programs. Emotional and behavioural dysregulation are key symptomatic expressions of vulnerability in children and important predictors of a broad range of mental outcomes during late adolescence and adult life [2]. The exploration of risk factors leading to this vulnerability represents an important area of research in child psychiatry. This issue of European Child and Adolescent Psychiatry (ECAP) includes several articles focusing on predictive factors for emotional and behavioural dysregulation in childhood or adolescence.

In one study, researchers analysed perceived parenting style, as measured by the Parental Bonding Inventory (PBI), as a potential factor modulating psychopathological outcomes in a school-based population of more than a thousand subjects [3]. The cohort was evaluated for emotional and behavioural difficulties at ages 7-9, and again 9 years later, at a mean age of 18 years. The authors found a high correlation between childhood emotional and behavioural traits and adolescent outcomes. They also found that perceived adequate maternal and paternal care had positive effects on socio-emotional development. Positive parent-child relationships had been regarded previously as enhancing resilience towards negative emotional and behavioural outcomes even in the presence of other environmental [4] or genetic risks [5]. However, in the present work, although optimal parenting of both parents was necessary for satisfactory emotional development, the authors found a differential effect. For participants who already displayed behavioural dysregulation during childhood, optimal care by the father,

Carmen Moreno

cmoreno@hggm.es

1 Child and Adolescent Psychiatry Department, Hospital General Universitario Gregorio Marañón, School of Medicine, Universidad Complutense, IiSGM, CIBERSAM, Madrid, Spain but not by the mother, lowered the risk of developing behavioural problems from pre-puberty to adolescence. This suggests that highly reactive children are especially sensitive to positive paternal care.

This ECAP issue also examines the biological underpinnings of emotional dysregulation. For example, van der Knaap et al. used functional MRI to study amygdala responses to negative emotional faces in children aged 6-9 years in the Generation R Study, a population-based sample [6]. They classified children by the presence of maternal depressive symptoms during pregnancy. Those with clinically relevant maternal depressive symptoms presented with increased amygdala hyper-responsivity, indicating neural processing different from that in control children. The findings do not establish the causality of psychopathology at the time of evaluation (6-9 years). In fact, the different groups were not significantly different in their frequency of internalizing or externalizing problems. However, most psychiatric disorders, including affective disorders, typically appear later in life, rendering the use of psychopathology to differentiate the two groups early on as problematic. Instead, the authors suggest that amygdala hyper-responsivity in children may be explored as a risk factor heightening the risk of future psychopathology. Daraki et al. investigated the association of maternal 25-hydroxyvitamin D $[25(\mathrm{OH}) \mathrm{D}]$ levels with multiple neurodevelopmental and cognitive outcomes at 4 years of age in a prospective pregnancy cohort including 487 mother-child pairs [7]. Low gestational maternal vitamin D levels had been previously linked to autism-related neurodevelopmental outcomes in population-representative samples [8]. In the new study, a broad range of behavioural problems and externalizing symptoms, including hyperactivity, impulsivity, and also inattention, were significantly lower in children born to mothers within the high $25(\mathrm{OH}) \mathrm{D}$ tertile $(>50.7 \mathrm{nmol} / \mathrm{l})$ as compared to offspring of women in the low $25(\mathrm{OH}) \mathrm{D}$ tertile $(<38.4 \mathrm{nmol} / \mathrm{l})$, supporting a protective effect of high maternal vitamin $D$ levels in early 
pregnancy on childhood behavioural outcomes, independent of cognitive factors.

Risk for future psychopathology is linked not only to the presence of risk factors, but also depends on the timing of risk factors. The aforementioned studies focus on prenatal factors. In fact, in the study of van der Knapp et al. prenatal depressive symptoms were related to altered amygdala functioning in the child, but postnatal maternal depressive symptoms 3 years after birth were not [6]. This suggests that genetic vulnerability alone is not enough to cause these neural processing changes; instead, symptoms must occur at a particular state of prenatal development. This underscores the relative importance of the intrauterine environment in the development of future psychopathology and environmental influence on the development of cortical structures critical to cognitive function and behavioural regulation. It also opens the possibility of considering this particular time frame as an opportunity for treatment. Animal studies have found that in offspring at high risk for neurodevelopmental disorders, administration of prenatal vitamin $\mathrm{D}$ prevented problematic behaviour [9]. This adds to the current knowledge on treatment of potentially environmentally based factors, such as treatment of maternal depression, which has also been found to positively impact child mental health outcomes [10], although consistent data on the effect of these interventions in pregnant women are lacking.

Studies including and following up on the offspring of parents with mental disorders provide a unique setting to study the interaction between genetic and environmental factors in non-clinically selected populations. Evidence supports the role of both genetic and epigenetic alterations as mediators of the intergenerational transmission of mental disorders [11]. Offspring studies also make it possible to monitor the prevalence and developmental changes of subtle expressions of psychopathology, including emotional and behavioural dysregulation, that are more prevalent among these children than in the general population [12]. The expression of psychopathology changes over time and also depends on pubertal status [12]. Although biological research is accruing [13] researchers have yet to validate with sufficient reliability biomarkers to serve as screening tools for prevention in younger and older offspring of parents with mental disorders. Longitudinal follow-up of these offspring, including biological measures such as neuroimaging and laboratory markers, seems critical to disentangle developmental psychopathology [14]. Besides, no validated preventive intervention programs are yet available for this high-risk population [14]. This is especially relevant in the case of younger children, who might be more amenable to interventions due to their enhanced neuroplasticity.

In spite of the above, mental health systems are not yet ready to provide an adequate, coordinated and flexible response for the offspring of parents with mental disorders.
It is a population that, despite its higher risk of psychopathology, does not always present with clear-cut psychiatric diagnoses. Instead, this population often suffers subtle and unspecific psychopathological manifestations and lower quality of life. Initiatives in this domain are rare, fragmented, and frequently linked to research rather than based on mental health settings. The medium and long-term economic impact of treatment strategies should be taken into consideration when designing services for children and adolescents. Mental health preventive interventions promoting early childhood development programs have been shown to be cost-effective [15]. High-risk offspring of parents with mental disorders constitute an ideal population for studying psychiatric risk factors and to translate research knowledge into clinical practice.

Acknowledgements Supported by the Spanish Ministry of Economy, Industry and Competitiveness. Instituto de Salud Carlos III (FIS PI17/02227 and PI14/02096), co-financed by ERDF Funds from the European Commission, "A way of making Europe", CIBERSAM. Madrid Regional Government (S2010/BMD-2422 AGES), European Union Structural Funds and European Union Seventh Framework Program and H2020 Program; Fundación Alicia Koplowitz and Fundación Mutua Madrileña.

\section{Compliance with ethical standards}

Conflict of interest Dr. Moreno has been a consultant for Janssen, Servier, Lundbeck, Otsuka and Nuvelution.

\section{References}

1. Lee FS, Heimer H, Giedd JN, Lein ES, Šestan N, Weinberger DR, Casey BJ (2014) Adolescent mental health-opportunity and obligation. Science 346(6209):547-549

2. Bertocci MA, Bebko G, Versace A, Fournier JC, Iyengar S, Olino T, Bonar L et al (2016) Predicting clinical outcome from reward circuitry function and white matter structure in behaviorally and emotionally dysregulated Routh. Mol Psychiatry 21:1194-1201

3. Ong MY, Eilander J, Saw SM, Xie Y, Meaney MJ, Broekman BFP (2018) The influence of perceived parenting styles on socio-emotional development from pre-puberty into puberty. Eur Child Adolescent Psychiatry. https://doi.org/10.1007/s00787-017-1016-9

4. Flouri E, Midouhas E, Joshi H, Tzavidis N (2015) Emotional and behavioural resilience to multiple risk exposure in early life: the role of parenting. Eur Child Adolesc Psychiatry 24(7):745-755. https://doi.org/10.1007/s00787-014-0619-7

5. Reupert A, Maybery D (2007) Families affected by parental mental illness: a multiperspective account of issues and interventions. Am J Orthopsychiatry 77(3):362-369

6. van der Knaap NJF, Klumpers F, El Marroun H, Mous S, Schubert D, Jaddoe V, Hofman A, Homberg JR, Tiemeier H, White T, Fernández G (2018) Maternal depressive symptoms during pregnancy are associated with amygdala hyperresponsivity in children. Eur Child Adolesc Psychiatry. https://doi.org/10.1007/ s00787-017-1015-x

7. Daraki V, Roumeliotaki T, Koutra K, Chalkiadaki G, Katrinaki M, Kyriklaki A, Kampouri M, Margetaki K, Vafeiadi M, Papavasiliou S, Kogevinas M, Chatzi L (2018) High maternal vitamin 
D levels in early pregnancy may protect against behavioral difficulties at preschool age: the Rhea mother-child cohort, Crete, Greece. Eur Child Adolesc Psychiatry. https://doi.org/10.1007/ s00787-017-1023-x

8. Vinkhuyzen AA, Eyles DW, Burne TH, Blanken LM, Kruithof CJ, Verhulst F, Jaddoe VW, Tiemeier H, McGrath JJ (2016) Gestational vitamin D deficiency and autism-related traits: the Generation R Study. Mol Psychiatry. https://doi.org/10.1038/ mp.2016.213 (Epub ahead of print)

9. Vuillermot S, Luan W, Meyer U, Eyles D (2017) Vitamin D treatment during pregnancy prevents autism-related phenotypes in a mouse model of maternal immune activation. Mol Autism 7(8):9. https://doi.org/10.1186/s13229-017-0125-0

10. Cuijpers P, Weitz E, Karyotaki E, Garber J, Andersson G (2015) The effects of psychological treatment of maternal depression on children and parental functioning: a meta-analysis. Eur Child Adolesc Psychiatry 24(2):237-245. https://doi.org/10.1007/s0078 7-014-0660-6

11. Slatkin M (2009) Epigenetic inheritance and the missing heritability problem. Genetics 182(3):845-850

12. Morón-Nozaleda MG, Díaz-Caneja CM, Rodríguez-Toscano E, Arango C, Castro-Fornieles J, de la Serna E, Espliego A,
Sanchez-Gistau V, Romero S, Baeza I, Sugranyes G, Moreno C, Moreno D (2017) A developmental approach to dimensional expression of psychopathology in child and adolescent offspring of parents with bipolar disorder. Eur Child Adolesc Psychiatry 26(10):1165-1175

13. Sugranyes G, de la Serna E, Borras R, Sanchez-Gistau V, Pariente JC, Romero S, Baeza I, Díaz-Caneja CM, Rodriguez-Toscano E, Moreno C, Bernardo M, Moreno D, Vieta E, Castro-Fornieles J (2017) Clinical, cognitive, and neuroimaging evidence of a neurodevelopmental continuum in offspring of probands with schizophrenia and bipolar disorder. Schizophr Bull 43(6):1208-1219

14. Benarous X, Mikita N, Goodman R, Stringaris A (2016) Distinct relationships between social aptitude and dimensions of maniclike symptoms in youth. Eur Child Adolesc Psychiatry 25(8):831842. https://doi.org/10.1007/s00787-015-0800-7

15. Zechmeister I, Kilian R, McDaid D, MHEEN group (2008) Is it worth investing in mental health promotion and prevention of mental illness? A systematic review of the evidence from economic evaluations. BMC Publ Health 22(8):20. https://doi. org/10.1186/1471-2458-8-20 